\title{
High speed imaging of traveling waves in a granular material during silo discharge
}

\author{
Tamás Börzsönyi1, ${ }^{1, *}$ and Zsolt Kovács ${ }^{1}$ \\ ${ }^{1}$ Research Institute for Solid State Physics and Optics, P.O. Box 49, H-1525 Budapest, Hungary
}

\begin{abstract}
We report experimental observations of sound waves in a granular material during resonant silo discharge called silo music. The grain motion was tracked by high speed imaging while the resonance of the silo was detected by accelerometers and acoustic methods. The grains do not oscillate in phase at neighboring vertical locations, but information propagates upward in this system in the form of sound waves. We show that the wave velocity is not constant throughout the silo, but considerably increases towards the lower end of the system, suggesting increased pressure in this region, where the flow changes from cylindrical to converging flow. In the upper part of the silo the wave velocity matches the sound velocity measured in the same material when standing (in the absence of flow). Grain oscillations show a stick-slip character only in the upper part of the silo.
\end{abstract}

When a granular material is slowly discharged from a vertically aligned container (tube) one often observes vibrations accompanied by a booming low frequency (typically $50-200 \mathrm{~Hz}$ ) sound. This phenomenon can be observed in large silos and is often referred to as silo music or silo honking, but it can also easily be reproduced in smaller scale laboratory experiments $1[-8]$. Silo quaking can also be observed during discharge [9, 10] where short sharp bursts of motion are separated by longer periods of no movement.

Although resonances during silo discharge are long known in industry and have recently been investigated thoroughly [1 4], the phenomenon is not yet fully understood. While one explanation is based on stick-slip motion of the grains near the silo walls [4 6 ], other authors argue that the phenomenon originates from a dynamic interaction between the silo structure and the flowing material, where the source of the vibration is the transition zone between cylindrical and converging flow in the lower part of the system near the outlet [1 3]. Recent laboratory experiments carefully investigated the frequency spectrum of the vibrations by piezoelectric accelerometers and microphones [ 1 [ 5$]$ and related these observations of the calculated eigenfrequencies of the silo structure [3] and the time evolution of the density of the material detected by a non-invasive method (Electrical Capacitance Tomography) [1, 2]. Optical imaging of silo quaking was reported in [11] using a frame rate of $8.3 \mathrm{fps}$. Here the predominant frequency of the bursts was about $1 \mathrm{~Hz}$, which was significantly lower (by a factor of 2 or 4 ) than the frequency of the silo wall vibrations indicating no resonance in this case. Moreover, grain oscillations were in phase over the largest part of the silo wall, so grain motion could not be linked to wave propagation. This contradicts other measurements on silo quaking [9, 10] where the quake propagated upwards in the silo with a well defined velocity and growing amplitude, as detected by accelerometers embedded into the granular material. Very recent observations 12] on resonant pipe flow (the pipe

${ }^{*}$ Electronic address: btamas@szfki.hu is continously fed from above) detected upward propagating waves using piezoelectric sensors, and claim constant wave speed in the entire tube, meaning, that the transition zone near the outlet has no special role in the formation of the resonance. This contradicts other measurements [3], where manipulating the geometry of the transition zone allows for very effective reduction of the resonance.

In this Brief Report we present direct observations of the grain motion during resonant silo discharge by means of high speed imaging using a frame rate of 3000 fps. This non-invasive technique allows us to better characterize the spatial evolution of the system. The aim of this study is to measure whether the grain oscillations show a stick-slip character near the walls, and if so, in which part of the silo? Are there regions in the silo, where the grains oscillate coherently in neighboring vertical positions, or not? What is the speed of wave propagation in the granular material during resonance?

In the present experiments the silo flow was realized in a vertically aligned glass tube of length $150 \mathrm{~cm}$ (see Fig. 1(a)). Two tubes were used with the inner diameters of

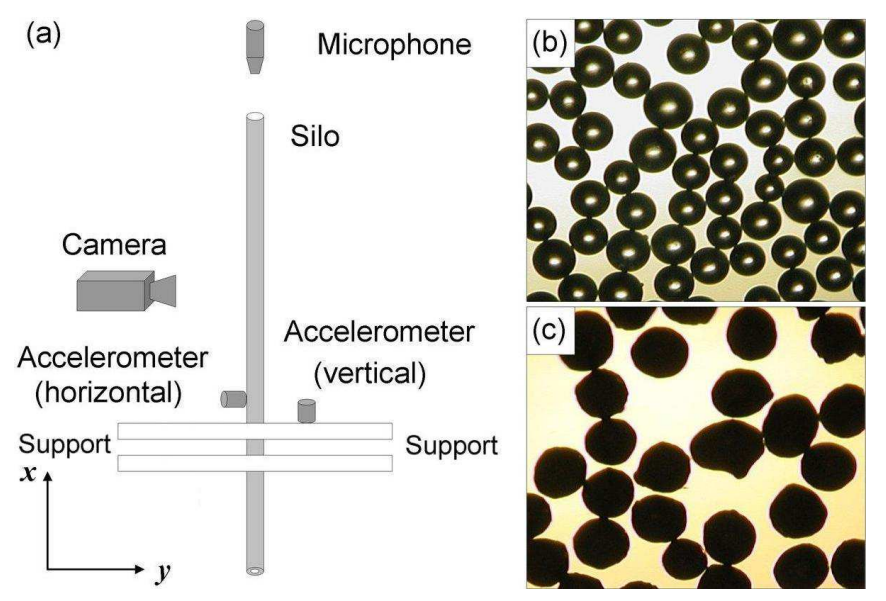

FIG. 1: (color online) (a) Schematic illustration of the experimental setup and (b)-(c) microscopic images of the materials used: (b) glass beads with mean diameter of $d=0.15 \pm 0.05$ $\mathrm{mm}$, (c) copper particles with $d=0.15 \pm 0.05 \mathrm{~mm}$. 
$D=3.55 \mathrm{~cm}$ and $2.3 \mathrm{~cm}$. We present results for two materials (i) nearly spherical copper particles with average grain diameter of $d=0.15 \pm 0.05 \mathrm{~mm}$ and (ii) spherical glass beads with $d=0.15 \pm 0.05 \mathrm{~mm}$ (see Figs. प(b)-(c)). Qualitatively similar results were obtained with quartz sand, granite and corundum of similar sizes, but in those cases the resonance was less pronounced. For the detection of the grain motion we used high speed cameras (Mikrotron CAMMC1310 and 1362) with a frame rate of $3000 \mathrm{fps}$. The resonance was also detected by traditional methods: piezoelectric accelerometers mounted on the glass tube (for horizontal vibration), on the support of the tube (for vertical vibration), and a condenser microphone with large diaphragm (IMG Stageline ECM-140). The silo discharge speed was varied by changing the diameter of the outlet $D_{\text {out }}$ at the bottom of the tube. As the main features of the phenomenon we are focusing on do not depend on the precise value of $D_{\text {out }}$, for convenience a constant value $D_{\text {out }}=8 \mathrm{~mm}$ was chosen for this study.

First we present the general features of the resonance obtained by piezoelectric accelerometers and the microphone. In one experiment the above signals have been recorded during the initially full hopper discharges. This
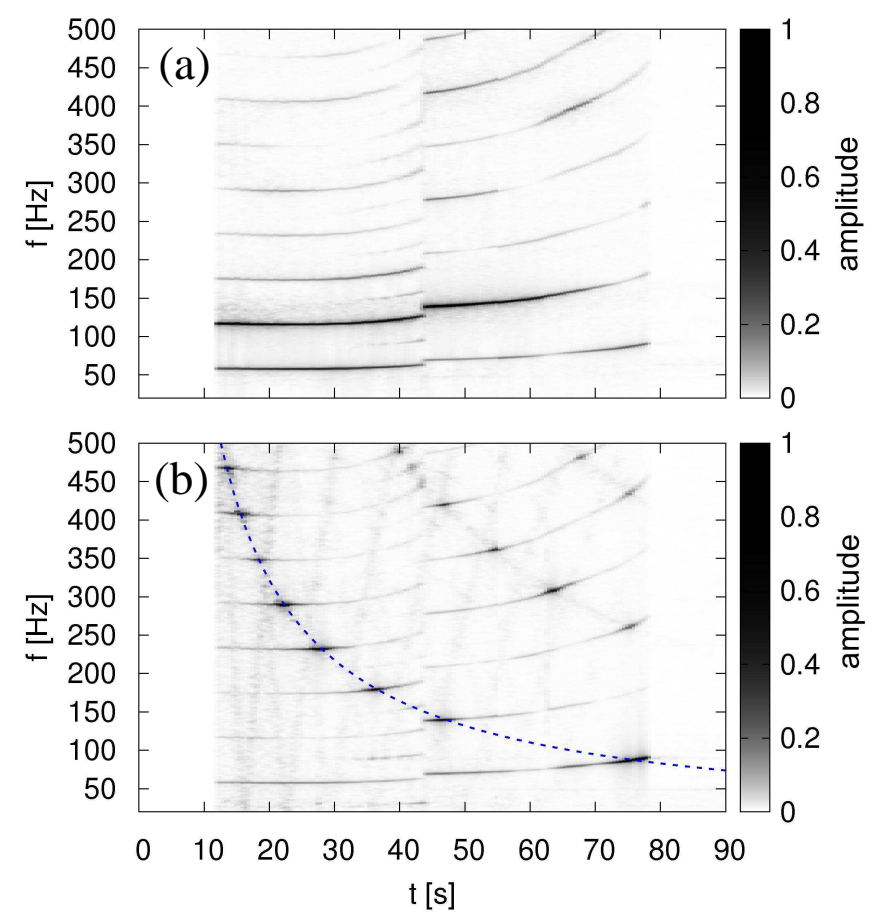

FIG. 2: (color online) Time evolution of the DFT power spectrum of the signals obtained by the (a) accelerometer mounted on the tube, (b) microphone. Data obtained with copper particles in the tube with the diameter of $D=3.55 \mathrm{~cm}$. The dashed line corresponds to the frequency of the standing wave (first harmonic) in the air column above the material.

takes about $80 \mathrm{~s}$ and $120 \mathrm{~s}$ for the tube with $D=2.3 \mathrm{~cm}$ and $D=3.55 \mathrm{~cm}$, respectively. The power spectrum of these signals has been calculated using discrete Fourier transform (DFT) with measurement windows of $0.5 \mathrm{~s}$ and is shown as a function of time in Figs. 2(a) and (b). As it is seen in Fig. 2(a) at the beginning of the process the system vibrates with a fundamental frequency of about $60 \mathrm{~Hz}$, and this frequency slightly increases as the silo discharges. At $t=44 \mathrm{~s}$ there is a quick transition characterized by a jump in the dominant frequency, but the trend of a slowly increasing frequency of the dominant mode persists until the resonance disappears at about $t=78 \mathrm{~s}$. This picture is fully coherent with other observations [1 $[3]$ and can be explained as follows. The granular flow excites the eigenmodes of mechanical vibration of the tube itself and the frequency of these modes is slightly increasing in time as the material is flowing out of the system and the weight of the oscillating body decreases. The time evolution of the system allows for changing the actually excited eigenmode, for which we have an example at $t=44 \mathrm{~s}$ where we see a jump in the dominant resonant frequency.

The signal picked up by the microphone shows the same characteristics as that of the accelerometer, but in addition to the eigenmode with slightly increasing frequency we find a resonance with decreasing frequency. This is the sign of standing sound waves in the air column in the tube above the granular material. As the length $l$ of this column is increasing with time (as the silo discharges) the frequency of the dominant mode is decreasing. The amplitude of this signal gets strong when it resonates with the "driving" frequency. The dashed line corresponds to the frequency of the standing wave (first harmonic) according to the formula of $f(t)=\frac{c}{\lambda(t)}=\frac{c}{4 l(t)}=\frac{1}{4} \cdot \frac{c}{v_{s} t+0.3 D}$ where $v_{s}$ is the velocity by which the top surface of the granular bed is sinking, $c$ is the velocity of sound in air and $0.3 D$ is the length correction for the open end of the tube [13]. The value of $v_{s}$ has been measured in an additional experiment, where the whole process was recorded optically and the position of the top surface was determined by digital image analysis. Several measurements confirmed, that $v_{s}$ is nearly independent of time except near the very end of the process where $v_{s}$ slightly increased.

In the following we will focus on the data obtained with high speed imaging. First we show how the grains move at a selected height $x$. For this we took images of a vertical stripe of $1 \times 0.1 \mathrm{~cm}$ and determined the displacement of the particles by correlating subsequent images. This procedure was repeated at various heights. In Fig. 3 three examples are shown, where Fig. 3(a) shows the position $s$ of the particles as a function of time, while (b) and (c) show the corresponding velocity $v$ and acceleration $a$. The three measurements correspond to $x=12$ $\mathrm{cm}, x=67 \mathrm{~cm}$ and $x=127 \mathrm{~cm}$ measured from the bottom of the tube. As it is seen, the grains move downwards with oscillating velocity. At the top and middle of the tube during each period they come to a full stop, remain standing for a considerable portion of the period and then accelerate downwards. Their acceleration almost reaches the value corresponding to free fall $\left(g=-9.81 \mathrm{~m} / \mathrm{s}^{2}\right)$ 
near the top of the tube, but is lower in the middle of the tube. At the bottom of the tube one can still see oscillations in the grain velocity but the grains do not
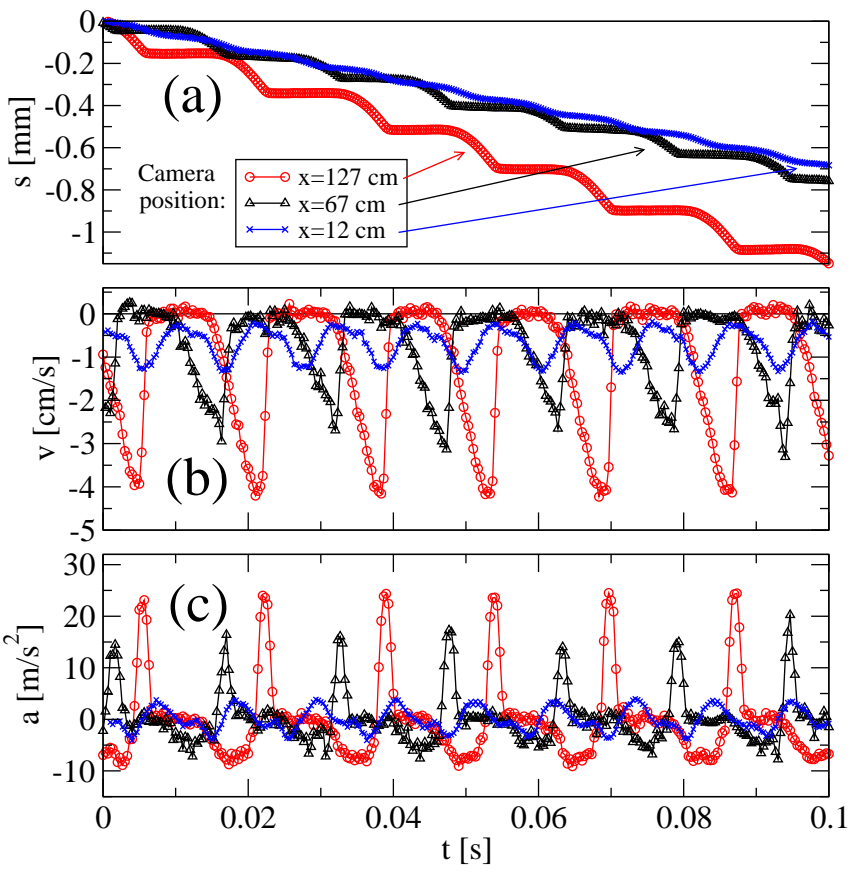

FIG. 3: (color online) (a) Grain position, (b) velocity and (c) acceleration as a function of time taken at three heights $x=12 \mathrm{~cm}(\times), x=67(\triangle) \mathrm{cm}$ and $x=127(\mathrm{\circ}) \mathrm{cm}$ measured from the bottom of the tube. Data taken with copper particles in the tube with the diameter of $D=3.55 \mathrm{~cm}$.

come to a full stop and their acceleration does not come close to $g$. The average grain velocity near the walls $v_{a v}$ (i.e. the slope of the curves in Fig. 3(a)) is smaller than the velocity $v_{s}$ by which the top surface of the granular bed is sinking. The corresponding three values are $v_{a v} / v_{s}=0.54,0.59$ and 0.89 for $x=12 \mathrm{~cm}, 67 \mathrm{~cm}$ and $127 \mathrm{~cm}$, respectively. This means that no perfect plug flow develops, i.e. the grains move slower near the walls than in the center of the tube even at the uppermost part of the material.

In order to measure whether or not the grains at different heights oscillate in phase with each other, a longer vertical stripe of $13.2 \times 0.5 \mathrm{~cm}$ was monitored. Two 2.2 $\mathrm{cm}$ long segments were analyzed at the upper and lower parts of this image. As it is seen in Figs. 4(a)-(b) there is a phase shift between these signals (indicated by vertical lines) implying that the grains in neighboring segments do not oscillate in phase with each other, but waves are propagating upwards in the system. The phase shift between the neighboring peaks in Fig. 4(a) corresponds to a wave velocity of $U=54 \mathrm{~m} / \mathrm{s}$, while in the lower part of the tube (Fig. 4(b)) a larger wave velocity (smaller phase shift) is observed. The velocity $U$ of the upward propagating waves has been measured at 6 different vertical positions. The resulting $U(x)$ curves are shown in figs.
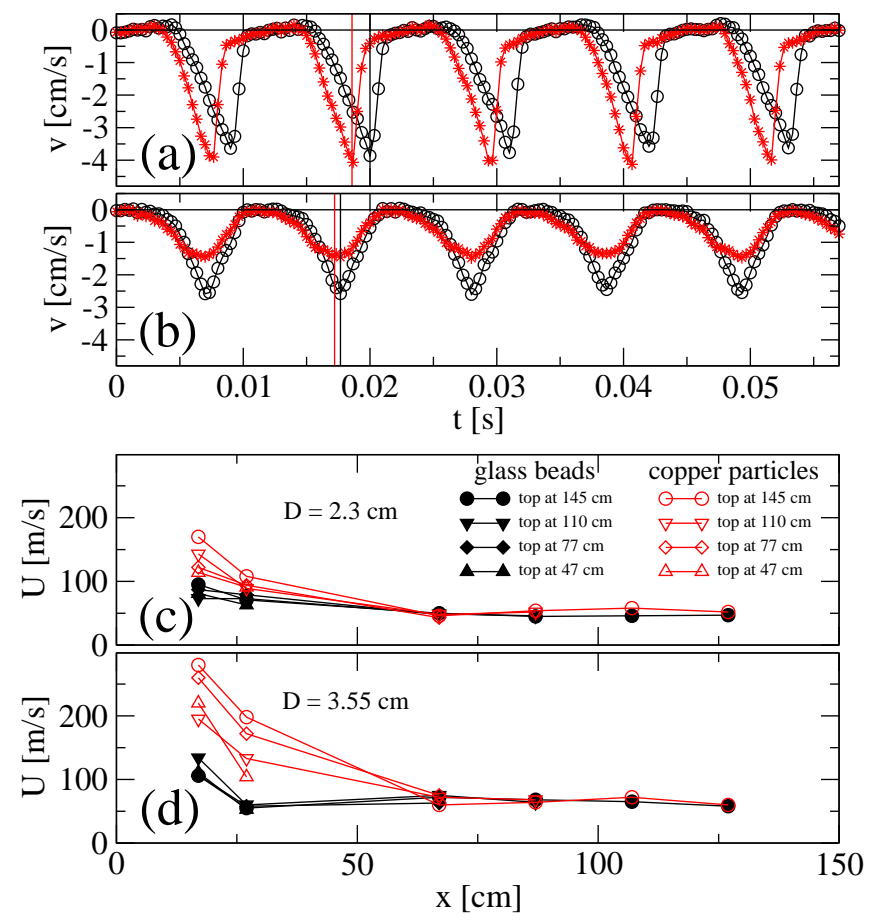

FIG. 4: (color online) Grain velocity $v$ as a function of time at two nearby locations at (a) $x=102 \mathrm{~cm}(\star)$ and $x=113$ $\mathrm{cm}(\mathrm{o})$ and (b) $x=22 \mathrm{~cm} \mathrm{( \star )} \mathrm{and} x=33 \mathrm{~cm} \mathrm{(o).} \mathrm{The}$ time lag between the curves is a measure of the corresponding sound velocity. (c)-(d) Velocity of sound waves as a function of location $x$ in the tube with (c) $D=2.3 \mathrm{~cm}$ and (d) $D=3.55$ $\mathrm{cm}$. The curves correspond to different stages of the process, when the top of the material was at $x=145 \mathrm{~cm} \mathrm{(o),} x=110$ $\mathrm{cm}(\nabla), x=77 \mathrm{~cm}(\diamond)$ and $x=47 \mathrm{~cm}(\triangle)$. Data for copper with open symbols, data for glass beads with solid symbols.

4(c) and (d) for the two tubes with $D=2.3 \mathrm{~cm}$ and 3.55 $\mathrm{cm}$, respectively. This measurement has been repeated at four different stages of the process, where the top of the material was at $x=145 \mathrm{~cm}, x=110 \mathrm{~cm}, x=77$ cm and $x=47 \mathrm{~cm}$. The wave velocity $U$ appears to be constant in the upper part of the tube. In the smaller tube $(D=2.3 \mathrm{~cm})$ we get $U=44 \pm 6 \mathrm{~m} / \mathrm{s}$ for glass beads and $U=54 \pm 6 \mathrm{~m} / \mathrm{s}$ for the copper particles, while in the larger tube $(D=3.55 \mathrm{~cm}) U=65 \pm 6 \mathrm{~m} / \mathrm{s}$ for both materials. These values are similar to those previously observed for the velocity of upward propagating pulses detected during "silo quaking" [9]. There single rarefaction waves (quakes) propagated upwards in the system with a velocity of $74 \pm 15 \mathrm{~m} / \mathrm{s}$ and $135 \pm 20 \mathrm{~m} / \mathrm{s}$ in the two Perspex tubes with the diameters of $9 \mathrm{~cm}$ and $30 \mathrm{~cm}$ respectively. The fact that we find larger value of $U$ in the larger tube also agrees with the above data obtained for quakes and is in accordance with very recent observations on continous pipe flow, where the velocity of sound waves was demonstrated to increase with increasing tube diameter [12]. Our more detailed spatial characterization however revealed (Figs. 4(c)-(d)), that the wave velocity is not constant throughout the tube but it increases 
towards the bottom of the tube. The larger value of $U$ measured at the lower part of the tube means a smaller phase lag between the oscillation of neighboring particles, which, in accordance with other results [1, 3], shows that the resonance originates from that part of the tube where the flow changes from cylindrical to converging flow (at $x \approx 4 D$ ) leading to strong density oscillations.

An additional experiment was done to measure the velocity of sound waves in the standing material (without flow) using the smaller tube $(D=2.3 \mathrm{~cm})$. In this measurement we placed 2 piezoelectric accelerometers in the granular material at a distance $d x$ above each other, and perturbed the granular bed at the lower end of the tube with a pneumatically generated single pulse. This was done using a smaller (flexible) tube with a rubber membrane at the end, which was placed in the lower end of the silo. We measured the time $d t$ needed for the pulse

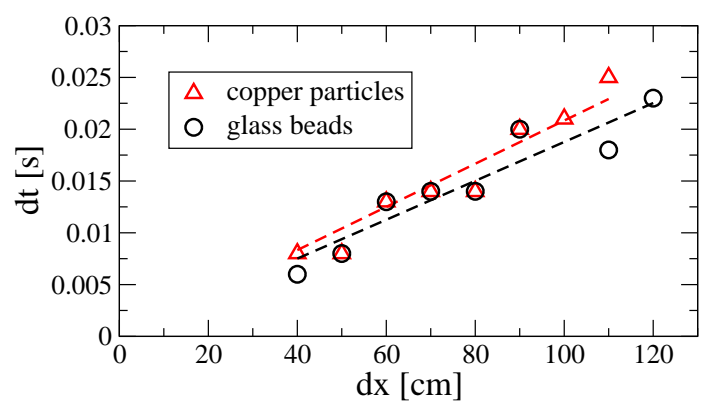

FIG. 5: (color online) The elapsed time $d t$ during a single pulse traveled between two piezoelectric accelerometers placed in the static material at a distance $d x$ for the case of copper $(\triangle)$ and glass beads (o) in the tube with $D=2.33 \mathrm{~cm}$. The slope of the linear fits (shown with dashed lines) gives $U_{s}$ the velocity of sound waves in the standing material.

to travel between the two accelerometers as a function of $d x$. The slope of the resulting curves (presented in Fig. 5) gives for the speed of sound waves in the standing material $U_{s}=53 \pm 5 \mathrm{~m} / \mathrm{s}$ for glass beads and $U_{s}=48 \pm 5$ $\mathrm{m} / \mathrm{s}$ for the copper particles. These values agree nicely with the ones discussed above observed in the same tube during flow (resonance) and are similar to other experimentally obtained values for the sound speed in glass beads $(60-240 \mathrm{~m} / \mathrm{s}$ [14]; $40-60 \mathrm{~m} / \mathrm{s}$ [16]) or sand $(40$ $\mathrm{m} / \mathrm{s}$ [15]), or even using much softer photoelastic particles $(100 \mathrm{~m} / \mathrm{s}$ [17]). These sound speed measurements were done in the absence of strong external load and also revealed, that the result can significantly depend on the measurement method [14]. More importantly, recent investigations uncovered, that in samples under external uniaxial load, the velocity of compressional waves is sensitive to stress induced anisotropy, leading to larger $U$ perpendicular to the applied load than parallel to it [19]. Also, $U$ increases more gradually with pressure $P$ $\left(U \propto P^{1 / 4}\right)$ at lower pressures, compared to the generally observed $U \propto P^{1 / 6}$ dependence at higher $P[18]$. Both of these observations [18, 19] indicate, that the larger wave velocity measured during resonant flow in the lower part of the tube suggests increased radial pressure in this region, where the velocity gradient changes due to the proximity of the outlet.

In summary, we studied a resonance phenomenon called "silo music" during silo discharge. We have visualized the oscillations in grain motion with high speed imaging, and detected that grain oscillations are not coherent when compared at different heights, but there is a phase lag. Information propagated upward in the system in the form of sound waves. One of our major findings is, that the wave velocity is not constant throughout the silo, but considerably increases towards the lower end of the system (corresponding to a more coherent motion of the grains). This underlines the importance of the transition zone in the lower end of the silo, where the flow changes from cylindrical to converging flow and is in accordance with those observations [1, [3] which suggest that the source of the resonance phenomenon is the generation of strong stress oscillations in this region. As shown, the amplitude of the grain oscillations increases with height and leads to stick-slip motion in the upper part of the silo. This supports the argument that appropriate friction with the walls is crucial for the amplification of the waves 9, 12, 20, 21 and could be a necessity for strong resonance as argued by other authors [4 6 ], which is also supported by the fact that increasing the roughness of the silo walls (i.e. suppressing stick-slip) effectively reduces the resonance [7, [8]. Finally, we have also shown that the velocity of the waves in the upper part of the silo matches the sound velocity measured in the same material when standing (in the absence of flow).

The authors are thankful for discussions with Kesava Rao, who motivated us to study this problem. We thank A. Konya for critical reading of this manuscript.
[1] K. Wilde, J. Tejchman, M. Rucka, M. Niedostatkiewicz, Powder Technology 198, 38 (2010).

[2] M. Niedostatkiewicz, J. Tejchman, Z. Chaniecki, K. Grudzien, Chemical Engineering Science 64, 20 (2009).

[3] K. Wilde, M. Rucka, J. Tejchman, Powder Technology 186, 113 (2008).

[4] M.L. Dhoriyani, K.K. Jonnalagadda, R.K. Kandikatla, K.K. Rao, Powder Technology 167, 55 (2006).
[5] B.K. Muite, F.S. Quinn, S. Sundaresan, K.K. Rao, Powder Technology 145, 190 (2004).

[6] J.M. Buick, J. Chavez-Sagarnaga, Z. Zhing, J.Y. Ooi, D.M. Pankaj, D.M. Cambell, C.A. Greated, Journal of Engineering Mechanics, ASCE 131, 299 (2005).

[7] J. Tejchman, Powder Technology 106, 7 (1999).

[8] J. Tejchman and G. Gudehus, Powder Technology 76, 201 (1993). 
[9] C. Wensrich, Powder Technology 127, 87 (2002).

[10] A.W. Roberts and C.M. Wensrich, Chem. Eng. Sci. 57, 295 (2002).

[11] J.M. Buick, Y. Pankai, J.Y. Ooi, J. Chavez-Sagarnaga, A. Pearce, G. Houghton, Journal of Physics. D: Applied Physics 37, 2751 (2004).

[12] L. Bonneau, T. Catelin-Jullien and B. Andreotti, Phys. Rev. E 82, 011309 (2010).

[13] J.W.S. Rayleigh, The Theory of Sound, Dover Publications (1945).

[14] C.H. Liu and S.R. Nagel, Phys. Rev. B 48, 15646 (1993);
C.H. Liu, Phys. Rev. B 50, 782 (1994).

[15] B. Andreotti, Phys. Rev. Lett. 93, 238001 (2004).

[16] L. Bonneau, B. Andreotti and E. Clement, Phys. Rev. Lett. 101, 118001 (2008).

[17] E.T. Owens and K.E. Daniels, unpublished.

[18] X. Jia, C. Caroli and B. Velicky, Phys. Rev. Lett. 82, 1863 (1999).

[19] Y. Khidas and X. Jia, Phys. Rev. E 81, 021303 (2010).

[20] C. Wensrich, Int. J. Mech. Sci. 45, 541 (2003).

[21] C. Wensrich, Powder Technology 126, 1 (2002). 